\title{
Ambiguity in a Dialectical Perspective
}

\section{Jan Albert van LaAR}

\section{Rijksuniversiteit Groningen}

\begin{abstract}
Résumé: On applique la distinction entre des règles constitutives et régulatives aux règles d'une discussion critique qui se rapporte à l'usage d'expressions ambiguës. Ceci mène à une distinction entre les sophismes qui contrevienment à des règles, dont la transgression entraîne la fin d'une discussion critique, et des sophismes qui contreviennent à des normes, dont la transgression est d'une certaine façon acceptable dans un discours critique. Selon le modèle formel d'une discussion critique qu'on propose, les transgressions sophistiques des normes sont recevables. D'ailleurs, ce modèle donne aux interlocuteurs des moyens de discuter des sophismes et des critiques de ceux-ci.
\end{abstract}

Keywords: rules for critical discussion, regulative rule, constitutive rule, rule violating fallacy, norm violating fallacy, point of order, ambiguity criticism, equivocation criticism, disqualified sentence, formal dialectics

\section{Introduction}

When playing soccer I have to compensate for my lack of skill and technique by making body checks and nasty tackles, which are offences against the rules. Now and then I am punished by a warning, or the other party gets compensated by obtaining a free kick. Although I am violating important and indispensable rules, my offences and the ensuing punishments are still part of what is properly called soccer. I am playing it in an incorrect way. If the rules are violated in a more drastic way however, for instance by closing the goal with an extra net in front of it, or by putting a second ball into the game, these actions are no longer part of what is properly called soccer. I am then not playing soccer anymore.

Having a discussion resembles playing soccer, in my view, in having these two kinds of rules. Consequently, in an adequate explication of the concept of having a discussion we should distinguish between on the one hand performing speech acts which are still discussion moves, but which are liable to some serious charge, and on the other hand performing speech acts by which one abandons what could really be called a discussion. 
Starting from this distinction, this paper examines the use of ambiguities in discussions. Which uses of ambiguity are still part of a critical discussion and can therefore be criticized within such a discussion, and which uses are not part of a critical discussion and can consequently not be criticized within a critical discussion?

To answer these questions, I shall first underscore the view that raising fallacy criticisms is an integral part of dialectics. Then I shall indicate what is wrong with contextual ambiguities but at the same time emphasize that it is very difficult, if not impossible, for real and imperfect discussants to steer clear of using contextually ambiguous expressions. This then is a reason for considering a reasonable procedure that constitutes a model for discussion that accomodates ambiguity. In the main part of the paper, I shall sketch such a procedure in an informal way. Incorporating fallacy criticisms gives rise to a classification of different types of fallacies, which will be provided at the end. In an appendix, a more formal description can be found of the type of dialogue presented in this paper.

\section{Capturing points of order: Ambiguous!, Equivocation!}

According to pragma-dialectics, discussions should ideally be regulated by a certain procedure for critical discussion. This procedure consists of rules which promote discussion moves that contribute to the main goal of a critical discussion, resolving a conflict of opinion. and moreover prohibit discussion moves that obstruct or hinder this goal. In case of a discussion aimed at resolving a difference of opinions, rule violations can and should be criticized as obstructing the goal of the discussion, that is, they should be considered as fallacious (van Eemeren $\&$ Grootendorst 1992, 104).

Suppose now that a proponent offers an argument that contains an expression that is ambiguous in the context of use, and consequently that it is not obvious what the intended interpretation is. Then the opponent can analyze the ambiguity and criticize the proponent for violating a rule for critical discussion. Rule 10 for critical discussion, which is at stake here, runs as follows: 'A party must not use formulations that are insufficiently clear or confusingly ambiguous and he must interpret the other party's formulations as carefully and accurately as possible' (van Eemeren \& Grootendorst 1992, p. 196). The proponent's contribution to the dialogue is not in accordance with the procedure for critical discussion and should therefore be withdrawn from the dialogue and possibly replaced by a non-ambiguous one. With the procedure as a regulative ideal, one is able to criticize bad discussion moves as fallacious. The pragma-dialectical procedure, however, does not regulate raising these fallacy criticisms. The reason probably is that the procedure describes ideal discussion behavior, and does not deal with how the rules are to be applied to non-ideal behavior, to rule-violations.'

Contrary to such an approach, Hamblin proposed to view the fallacy of equivocation from the perspective of making points of order ${ }^{2}$ within a discussion (1970). 
In interesting cases of ambiguity, theorists cannot determine whether in fact a fallacy has been committed or not. But they can view the situation from a dialectical perspective and investigate discussion moves in which one party accuses the other party of committing the fallacy of equivocation.

Mackenzie elaborated on this idea of making points of order. He proposed a formal dialectical system in which the opponent has a device to criticize an equivocal argument (1988). ${ }^{3}$ Moreover, he proposed a formal dialectical system that is made up of two parts. There is an inner system DC of rules that state which moves are DC-legal. If some party violates a DC-rule, there is a system of $\mathrm{DC}^{+}$ rules by which this violation can be criticized (1981, p. 172-176). ${ }^{4}$

Krabbe took up the issue from the angle of dialogue profiles (1996). Given a certain history of a discussion up to a point, the discussant whose move it is can choose from a set of alternatives. Some of these options concern topic points, other concern points of order. Among the options, Krabbe mentions active criticisms and fallacy criticisms. The purport of an active criticism is that an argument offered by the other party is wrong, mistaken or insufficient. The purport of a fallacy criticism is that the argument is inadmissible. Only fallacy criticisms are considered points of order by Krabbe.

In my view, including the option of making points of order contributes to the normative force and the empirical adequacy of models for critical discussion. Raising a point of order, for instance some kind of ambiguity criticism, can itself be seen as a contribution to the resolution of the dispute, and in that light as a good or a bad contribution. An ambiguity criticism is good if it detects a real ambiguity, forces the speaker to retract the ambiguous assertion, and gets the topical discussion in an efficient way back on a right track. So, good ambiguity criticisms contribute to conflict resolution. And an ambiguity criticism that is inappropriate, for instance because there is no ambiguity that is of any consequence to the course of the dialogue, is a bad contribution, precisely because it is an unfruitful digression that is a detour and because it can lead to a premature closure of the discussion. In section 5, I shall maintain that, when the issue of ambiguity arises, discussants can react in an adequate way by choosing from two different but related types of fallacy criticism. They need to be able to criticize something as a fallacy of ambiguity, and in addition they can criticize something as a fallacy of equivocation.

This view suggests the task of developing additional normative dialectical models for situations that are not optimal because of the use of ambiguous expressions. Given that we are sometimes not capable of avoiding ambiguities, the dialectics for less than optimal situations describes the best way to proceed. In order to capture points of order, the model should be constituted by rules that do not exclude that discussants violate norms for critical discussion and that enable them to repair these violations. Such a model would be an extension of the pragma-dialectical theory and a contribution to the tradition of theorists who emphasize points of 
order (Hamblin, Mackenzie), active criticisms (Finocchiaro 1980) and fallacy criticisms (Krabbe 1996) as integral parts of dialectics.

My point is two-sided. On the one hand, I want to maintain that ambiguity is worthy of being criticized as fallacious. On the other hand, I also want to maintain that, in a way, ambiguity is admissible in a critical discussion.

\section{Ideally, discussants do not use contextually ambiguous sentences.}

In order to be able to construct reasonable rules for using or criticizing ambiguities, we have to know what is or is not wrong with using ambiguous expressions in a discussion aimed at conflict resolution. It is my contention that using contextual ambiguity in an argument resembles a failure to argue ex concessis.

It is a commonplace to say that ambiguity per se is not wrong at all. We are quite often able to communicate successfully while using sentences that are ambiguous in the sense that they mean one thing in the current context of use, and would mean quite a different thing if used in another context. This kind of ambiguity does not concern us here. In line with, for instance, Walton $(1996,22)$, we focus on ambiguity of a contextual nature: the use of semantically ambiguous sentences whose ambiguity is not easily resolved by the context of use.

This paper is restricted to a simple kind of discussion, where there is a proponent who is committed to one main thesis and an opponent who doubts whether this thesis is acceptable. The proponent attempts to persuade the opponent of the view that, given her other commitments, she is committed to the proposition expressed in the main thesis. The opponent attempts to persuade the proponent of the view that her doubt regarding the main thesis is consistent with her overall position.

To enable each other to make a serious attempt at persuasion, the discussants should cooperate by making their position clear, that is by making clear to what they are actually committed. The proponent has to know to what the opponent is committed in order to be able to figure out what arguments might have success. The opponent has to know to what thesis the proponent is committed, or by what arguments the proponent is trying to persuade her, in order to know whether she can coherently refuse to accept the thesis or the reasons that are given. The scope of this paper is further restricted by considering only the proponent's use of ambiguities.

The propositional content of a commitment is communicated by sentences. Now suppose that the proponent makes an assertion by uttering a sentence $S$ in some context, that the opponent is in this context disposed to distinguish more than one reading of $S$, that these readings are not ruled out by the semantics and that the opponent is in this context not able to choose one of these readings as the right one. In that case $\mathrm{S}$ is ambiguous for the opponent. ${ }^{6}$ If the opponent is maximally attentive, then it is clear to her that the proponent has committed himself to at least one of these propositions. Asserting an ambiguous sentence gives some informa- 
tion about the position of the speaker, but does probably not provide a sufficient basis for the opponent to develop a critical position. The speaker's choice of a sentence $\mathrm{S}$ as a vehicle to express a proposition in this context does not match the listener's disposition to interpret $S$ in this context. If $S$ functions as part of an argument, the proponent's choice of words resembles a failure to argue ex concessis. Just as in the case of a failure to argue ex concessis, making an assertion by a sentence that is ambiguous for the opponent is a failure to adjust the argument to the position of the opponent: the proponent does not tailor the argument to the semantic dispositions of the opponent.

The most likely way to get such a defect repaired is by asking for a disambiguation of the argument. $A$ sentence $S$ is a less ambiguous reformulation of sentence $\mathrm{T}$ in context $\mathrm{C}$ if and only if all propositions expressed by $\mathrm{S}$ in $\mathrm{C}$ are also expressed by $\mathrm{T}$ in $\mathrm{C}$, and if there is at least one proposition expressed by $\mathrm{T}$ in $\mathrm{C}$ that is not expressed by $\mathrm{S}$ in $\mathrm{C}$. This definition is a modified version of Naess's definition of what he named in Norwegian 'en presisering' (Naess, 1966, 39). But we will consider these reformulations from a functional perspective, according to which $\mathrm{S}$ is a disambiguating reformulation of $\mathrm{T}$ in $\mathrm{C}$ if it is presented by a discussant as having the function of being a less ambiguous reformulation of $T$ in $\mathrm{C}$ (whether

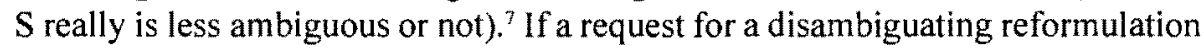
is answered in an appropriate way, then the position of the speaker becomes clearer to the listener.

Suppose the proponent makes an assertion by uttering 'Stopping before a traffic light is obligatory' and the opponent distinguishes in this context between two distinct propositions that can in this context be expressed non-ambiguously as 'Stopping before a traffic light is morally obligatory' and 'Stopping before a traffic light is legally obligatory'. According to the opponent then, the proponent committed himself to either the first or to the second proposition. If the proponent makes his intention clear by using one of these disambiguating reformulations, the opponent's uncertainty with regard to the proponent's position disappears.

As an ambiguity is often difficult to detect and as it is even more difficult to give an adequate analysis of it, there is a chance that the opponent does not see the weakness (or fallaciousness) of the proponent's argument or is not able to respond adequately. There are two specific risks involved.

First, an unidentified ambiguity can lead to a misunderstanding, where the speaker thinks she made it clear by uttering sentence $S$ that she expressed one proposition, and the listener supposes that another proposition is naturally expressed by the speaker's use of S. This again can lead to pseudo-agreement or pseudo-disagreement (Naess 1966, 83-96).

Secondly, the expression can be the source of what is known as the fallacy of equivocation. Viewed from a dialectical perspective, a proponent commits a fallacy of equivocation against an opponent, if (1) the opponent can distinguish between several readings of the argument, (2) there is at least one reading in which 
all reasons are acceptable to the opponent and there is at least one other reading in which the warrant is acceptable to the opponent, and (3) the argument fails, whatever reading the proponent would choose, either because a warrant is in the end not acceptable to the opponent, or one of the reasons offered in the argument is in the end not acceptable to the opponent. In Walton's terms, the argument has either bite or bearing, but not both (Walton 1996, 19). If the ambiguity goes unnoticed, there is the risk that the opponent is misled by the appearance of soundness and fails to detect good opportunities to criticize the argument and for that reason loses the discussion needlessly.

Using an ambiguous sentence can lead to misunderstanding or to a situation where the opponent does not see the opportunities to criticize the argument. If a sentence used by the proponent is contextually ambiguous, I say the proponent has committed the fallacy of ambiguity. So, according to my usage, committing a fallacy of equivocation entails committing a fallacy of ambiguity, but not viceversa.

So ideally, discussants in a critical discussion do not use any contextually ambiguous sentences. However, the harmful effects of using ambiguous or equivocal arguments are not in all situations equally serious. Suppose the proponent asserts an ambiguous sentence $S$. Then the proponent's use of $S$ does not match the opponent's dispositions to interpret S. But suppose further that the opponent had not yet made her semantic dispositions overt. After that, the opponent points at the ambiguity and the proponent repairs his position by using disambiguating reformulations. In such a situation, the discussants are at every stage working up to resolution. Although the discussion started with some unclearness, this is cleared up during the discussion. If no other obstacles arise, every move brings the discussants closer towards conflict resolution. Such discussion fragments are in my view part of a critical discussion.

But suppose that it was already clear in the discussion that, in the opponent's view, $\mathrm{S}$ is ambiguous. ${ }^{8}$ Then in a way, $\mathrm{S}$ is disqualified in this discussion as contextually ambiguous: it is an overt fact that the opponent perceives $\mathrm{S}$ as ambiguous. If the proponent nonetheless asserts $S$, this move brings them further away from conflict resolution. Such a move is, in my view, not a part of a critical discussion.

\section{Unfortunately, we do use ambiguous expressions.}

Whether sentence $S$ is ambiguous for the opponent depends on, among other things, whether the opponent can distinguish between different readings of S. If the proponent has a wrong picture of the opponent's semantic dispositions this may lead to the situation where the proponent makes an assertion by uttering $S$, while $S$ is ambiguous for the opponent and while the proponent did not intend to commit a fallacy of ambiguity. Whether sentence $S$ is the source of the fallacy of equivocation depends on S's being ambiguous and on the lack of an effective 
persuasion strategy for the proponent after disambiguating the occurrences of $\mathrm{S}$. So, if the proponent has an adequate picture of the opponent's semantic dispositions, even then it is possible that he commits a fallacy of equivocation unintentionally, due to a wrong picture of the opponent's (normal) commitments. ${ }^{9}$ As it is not in all situations feasible for the proponent to have an adequate picture of the opponent's position, it is not reasonable to prohibit and exclude the use of contextually ambiguous sentences.

An overview of different types of wrong pictures is given in the next figure.

The proponent starts from a wrong picture of the opponent's position



The proponent has a wrong picture of the opponent's (normal) concessions

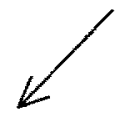

(1) The proponent has a wrong picture of what reasons the opponent will be prepared to concede

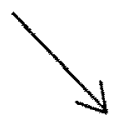

(2) The proponent has a wrong picture of what warrants the opponent will be prepared to concede

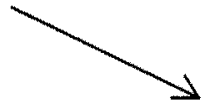

The proponent has a wrong picture of the opponent's dispositions to interpret a sentence

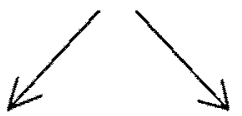

(3) The

(4) The proponent has an incomplete picture of the set of readings of a sentence that the opponent distinguishes proponent has an incorrect picture of the set of readings of a sentence that the opponent distinguishes $^{10}$

Figure 1: Wrong pictures underlying the fallacy of ambiguity and the fallacy of equivocation

The following examples illustrate that, for different reasons, we are not always able to avoid the use of contextually ambiguous sentences. An arrested person, W.B., describes the course of events of the night on which he was arrested for two offences. He supposedly drove a car, while his license was suspended and after he had drunk too much beer:"1

That evening, I left home for a visit to an acquaintance, I asked a friend to drive me [in my own car], for the judge had suspended my license. During the evening this friend left, so he couldn't drive me home. But my car wasn't safe there in front of this acquaintance's house. I couldn't leave my car where it 
was. So I decided to bring my car to a safe place - -a parking lot a little further on. I wasn't allowed to drive, and because of that I did, while walking, push the car, with its motor running, in the direction of the parking lot. I was arrested and I had drunk some beer. (Dutch jurisdiction: NJ 1991/29)

Now, I imagine that the following discussions took place, the first one while W.B. pushed his car to the parking lot, the second one while the issue was discussed in court.

\section{Discussion 1:}

Police officer, who had just arrived: Your license, please.

W.B.: Well, there is no license. But I am not driving a car, so I will not get convicted.

Officer: You are right that you are not driving a car, but only in the stricter sense of 'driving'. Your thesis, however, only follows from a premise that states that you are not driving a car in the legal sense, which is a wider sense.

\section{Discussion 2:}

W.B. 's defending counsel: W.B. should not be convicted, for he did not drive a car and only if he did drive a car, are there grounds to convict him.

Judge: W.B. did not drive a car in the stricter sense, but he did drive a car in the wider sense. And 'to drive', as it occurs in the relevant articles, is to be taken in the wider, encompassing sense.

Presumably, in discussion 1, W.B. starts from the wrong supposition that the police officer sees just one reading of the sentence 'I am not driving a car'. W.B. reckons only with a stricter sense of driving. Further, W.B. wrongly supposed that the police officer would accept the warrant 'if W.B. is not driving a car (in this strict sense) then W.B. will not get convicted'. So this is an example of a situation (type 3) where a proponent starts from an incomplete picture of the opponent's dispositions to interpret a sentence, as well as of a situation (type 2) where a proponent starts from a wrong picture of what warrants the opponent is prepared to concede.

The second discussion illustrates the situation where the defending counsel probably suspects there is an ambiguity, but wrongly thinks there is a disambiguation of his argument in which the reason along with the warrant is acceptable to the judge. The next figure shows that this constitutes an example of the situation (type 2) where the proponent has a wrong picture of what warrants the opponent is prepared to concede.

We are not always able to avoid ambiguous assertions and equivocal arguments if the ambiguities are not explicitly disqualified. The underlying ground is that we are not able to have a completely accurate and complete picture of the position of our opponents. As we are not always able to avoid fallacies of ambigu- 


\begin{tabular}{|c|l|l|}
\hline $\begin{array}{c}\text { disambiguated reason or } \\
\text { warrant }\end{array}$ & $\begin{array}{l}\text { defending counsel's } \\
\text { picture of what the } \\
\text { judge will accept }\end{array}$ & $\begin{array}{l}\text { real picture of } \\
\text { what the judge } \\
\text { will accept }\end{array}$ \\
\hline $\begin{array}{l}\text { W.B. did not drive a car } \\
\text { (in the stricter sense). }\end{array}$ & acceptable & acceptable \\
\hline $\begin{array}{l}\text { Only if W.B. did drive a car } \\
\text { (in the stricter sense) are } \\
\text { there grounds to convict him. }\end{array}$ & acceptable & not acceptable \\
\hline $\begin{array}{l}\text { W.B. did not drive a car } \\
\text { (in the wider sense). }\end{array}$ & not acceptable & not acceptable \\
\hline $\begin{array}{l}\text { Only if W.B. did drive a car } \\
\text { (in the wider sense), are } \\
\text { there grounds to convict him. }\end{array}$ & acceptable & acceptable \\
\hline
\end{tabular}

Figure 2: An example of a wrong picture that underlies the act of committing the fallacy of equivocation.

ity or fallacies of equivocation, it is not reasonable to exclude the use of contextual ambiguities from persuasion dialogues. Besides, our imperfections indicate the need for a dialectical procedure for making a point of order against the use of ambiguity.

\section{An optimal procedure for sub-optimal situations}

In the model I propose, named Ambiguity Dialectics (AD), there is a distinction between norms for critical discussion and rules for critical discussion. ${ }^{13}$ Norms for critical discussion indicate an optimal way of resolving disputes and are regulative (Searle 1970,33) for AD-discussions. In AD there is only one norm: 'Do not make assertions by uttering contextually ambiguous sentences!'. ${ }^{14}$ The AD-rules are the constitutive rules (Searle 1970,33) for AD-discussions. When in a dialogue an $\mathrm{AD}$-rule is violated, the dialogue cannot be called an $\mathrm{AD}$-discussion in the proper sense. However, it is possible to violate the AD-norm within an AD-discussion. In my view, the informal notion of a serious and critical discussion or a persuasion dialogue is best modeled by something like $\mathrm{AD}$, having both restrictive norms and more permissive rules.

Norms should promote the most efficient ways to discuss the topic under consideration. In order to keep themselves on an efficient track towards resolution, discussants need to have the means at their disposal to criticize any move in 
which a norm is violated. As the discussions under consideration are asymmetric, and only the proponent is able to assert topical points, only the opponent of the main thesis needs a device to criticize ambiguous assertions or ambiguous arguments. ${ }^{15}$ The AD-rules should, among other things, promote efficient ways to discuss points of order, active criticisms or fallacy criticisms.

According to the $\mathrm{AD}$-rules, the opponent is able to raise a fallacy criticism (as I will call it) only by making a statement of an assertive nature: you have made this particular move and by doing it you violated the norm!, by which she takes on a burden of proof. So, although the discussion is asymmetric in the sense that one party remains proponent of the main thesis while the other party remains the opponent of the main thesis, it must be possible for a shift of roles to occur after which the opponent of the main thesis becomes the proponent of a fallacy criticism. From now on I shall refer to the critic of the main thesis as "White" and its proponent as "Black".

Desiderata of the procedure are that ambiguous assertions (relative to the opponent) are withdrawn and replaced by non-ambiguous ones, that inappropriate fallacy criticisms are withdrawn, and that requests for disambiguations are used as sparsely as possible.

Opponents need to be able to criticize an ambiguous assertion by raising an ambiguity criticism. Raising such a criticism is an effective reaction to the threat of misunderstanding and to alleged instances of the fallacy of equivocation. A standard way to criticize specific uses of ambiguity is by raising an equivocation criticism. This is an effective reaction to an alleged fallacy of equivocation, but is a device too heavy for most threats of misunderstanding. Equivocation criticism is therefore less basic than ambiguity criticism.

A standard formulation of ambiguity criticism could be the following:

You have made an assertion using sentence $S$ while in this context $S$ can express $n$ different propositions. ${ }^{16}$

White should have a burden of proof for this. First of all, she should retract such an ambiguity criticism if Black did not really use sentence $S$. But a discussion on this point is not very interesting, so there must be a constitutive rule to the effect that White can raise an ambiguity criticism against the use of a sentence $S$ only if Black actually has uttered S. Second, S must express $n$ propositions. The most natural way for Black to criticize this aspect is to ask 'Which ones?'. White then will have to present $n$ different formulations for $n$ different propositions. To speed up the dialogue, I have made this particular defense an integral part of the standard formulation of criticizing ambiguity. 
Ambiguity criticism:

You have made an assertion using sentence $S$ while in this context of use $\mathrm{S}$ can express $n$ different propositions, and these propositions can, for this context, be adequately formulated as $S_{1}, \ldots, S_{n}$.

Black should be able to investigate whether in this context $\mathrm{S}$ is ambiguous between $S_{1}, \ldots, S_{n}$ by testing whether the semantics of their language really admits all $S_{i}$ 's as proper disambiguating reformulations in this context. Since this is not a study in semantics, I imagine that the discussants have some sort of utterance meaning machine at their disposal by which they can perform an utterance meaning testing procedure. ${ }^{17}$ This is inspired by other types of testing procedures (van Eemeren en Grootendorst 1992, 158). The machine enables the discussants to test whether White's linguistic analysis is coherent with the linguistic conventions of the language White and Black have adopted.

I presuppose that at least for some combinations of supposedly ambiguous sentences $\mathrm{S}$, disambiguating reformulations $\mathrm{S}_{1}, \ldots, \mathrm{S}_{\mathrm{n}}$ and contexts $\mathrm{C}$, it can be linguistically determined whether or not each $S_{i}$ expresses a proposition in $C$ that $S$ does also express in $\mathrm{C}$. These linguistic data should constrain the discussants' options. It is also realistic to assume, however, that there are combinations of supposedly ambiguous sentences, disambiguating reformulations and contexts, for which the shared language does not give a clear result. In such a case $S$ is ambiguous in a more subtle way. The 'W.B.' example illustrates the case where the language does not determine whether or not a term (and consequently sentences in which the term occurs) is ambiguous between two senses, but where the discussants have to decide by other means how to continue the discussion. ${ }^{18}$

The utterance meaning machine is supposed to work as follows. Black puts a combination of $S$ (the focus of White's fallacy criticism), $S_{1}, \ldots, S_{n}$ (the disambiguated reformulations offered by White) and information about the context of use into the machine. The machine answers 'negative' or 'positive'. The negative outcome means that two or more $\mathrm{S}_{i}$ 's mean in this context exactly the same, or that some $\mathrm{S}_{i}$ in this context does not express anything that $S$ expresses in this context. The positive outcome means just that the outcome is not negative. This is the outcome, for example, if ( 1 ) every $S_{i}$ in this context means something else and every $S_{\text {i }}$ expresses a proposition in this context that $S$ in this context also expresses, or if (2) there is at least one $\mathrm{S}_{i}$ for which it holds that the language does not determine whether or not it expresses a proposition that $\mathrm{S}$ does in $\mathrm{C}$, while there is no $\mathrm{S}_{\mathrm{j}}$ for which it holds that the language precludes it as a proper disambiguating reformulation. If the test-outcome is negative, White has to retract her ambiguity criticism. If the test-outcome is positive, Black is obliged to accept the ambiguity criticism.

This use of the machine entails that White's semantic dispositions are favored over those of Black in case the issue cannot be determined on the basis of the semantics. This reflects the idea that Black has to tailor the attempt at persuasion 
to the position of White, also when it comes to semantic issues, but that the common language restricts White's freedom with regard to semantic issues.

After an ambiguity criticism, Black is allowed, and after a positive test-outcome even obliged, to accept the criticism. Black accepts it by offering a disambiguated argument, that is, Black's complete argument, as given until that stage of the discussion, in which each occurrence of $\mathrm{S}$ is disambiguated.

One way for Black to disambiguate his argument is by replacing each occurrence of $S$ by either $S_{1}$ or ... or $S_{n}$. White has determined what formulations are appropriate, Black decides what assertions to make with them. This way Black repairs his violation of the norm and the discussion is back on track (as viewed by the discussants themselves). White should, as she is herself responsible for the adequacy of the formulations $S_{1}, \ldots, S_{n}$, not be allowed to raise an ambiguity criticism to one of these $S_{i}$ 's. This restricts her freedom to criticize formulations as ambiguous.

Yet another way for Black to disambiguate his argument is by replacing occurrences of $S$ by disambiguated reformulations $S_{1}^{\prime}, \ldots, S_{m}{ }_{m}$ that do not all occur in White's fallacy criticism. In this case, Black accepts that there is some ambiguity, but gives a (partly) different semantic analysis of it. This option for Black is needed, since White may distinguish semantically appropriate readings of $S$, but not all admissible readings that Black considers relevant for his defense.

After such a move the sentences $S_{i}^{\prime}$ that were not offered by White as adequate disambiguating reformulations of $S$ must themselves be liable to ambiguity criticisms. Furthermore, the combination of $\mathrm{S}$ and $\mathrm{S}_{1}^{\prime}, \ldots, \mathrm{S}_{\mathrm{m}}^{\prime}$, together with the current context, must be liable to an utterance meaning testing procedure performed by White. When the outcome is positive, White must accept the reformulations as proper disambiguations ${ }^{19}$ (although she may still challenge the acceptability of reasons and warrants after a substitution takes place). When the outcome is negative Black must replace all occurrences of $S$ by $S_{1}, \ldots, S_{n}$, the disambiguating reformulations chosen by White.

I propose the following standard formulation for the fallacy of equivocation criticism:

You have made an assertion using sentence $\mathrm{S}$, while in this context $\mathrm{S}$ can express $n$ different propositions, and these propositions can be, for this context, adequately formulated as $S_{1}, \ldots, S_{n}$, and furthermore, 1 shall win the discussion whatever disambiguated argument you will come up with.

Black can react in the same ways as when White raised a simple ambiguity criticism. But White's burden of proof for an equivocation criticism is heavier. White should retract her equivocation criticism if the test-outcome is negative, but also if Black accepts the ambiguity criticism that is part of the equivocation criticism, consequently disambiguates his argument and wins the ensuing dialogue. By presenting a disambiguating argument Black challenges White's equivocation criti- 
cism. If White concedes Black's main thesis at some point after her equivocation criticism, her criticism is regarded as withdrawn. Black accepts an equivocation criticism only by retracting his thesis.

After White has raised an ambiguity or equivocation criticism and until she retracts this criticism of sentence $S$, Black should not be allowed to make an assertion by uttering sentence $\mathrm{S}$. During these stages, $\mathrm{S}$ is a disqualified sentence.

Until now, we considered a procedure for how to behave in case a norm has been violated. Within an AD discussion, there can be no such procedure for violations of the rules, for they constitute such discussions. But probably there is a reasonable procedure for rule-violations within other types of dialogue (Walton \& Krabbe 1995, Chapter 3).

\section{Is ambiguity fallacious or not?}

In $\mathrm{AD}$, the norms are part of a procedure for resolving conflicts in an optimal way. In a pragma-dialectical sense, violations of $\mathrm{AD}$-norms are therefore fallacies. I shall call these Norm Violating Fallacies or $N$-fallacies. These moves deserve to be actively criticized as an obstacle to the resolution of the initial conflict of opinions, although they are a proper part of a discussion. $\mathrm{N}$-fallacies are not ruled out by the rules of $\mathrm{AD}$. I call violations of AD-rules Rule Violating Fallacies, or $R$ fallacies. These are more serious obstacles to conflict resolution. By committing an R-fallacy, the perpetrator has abandoned the confines of a critical discussion altogether. ${ }^{20}$

Whether some sentence $S$ is contextually ambiguous or not depends on White's language-constrained choices or dispositions to interpret $\mathrm{S}$. Whether such an ambiguity constitutes a $\mathrm{N}$-fallacy or a R-fallacy depends on whether $\mathrm{S}$ is or is not disqualified at the stage of Black's utterance of $S$. Whether $S$ is part of a fallacy of equivocation depends on whether Black has an effective persuasion strategy for his main thesis after presenting his disambiguated argument in which $\mathrm{S}$ is replaced by disambiguating reformulations of S. Contrary to Walton $(1996,72)$, I think there is a need for having the concept of the fallacy of (contextual) ambiguity, besides a concept of the fallacy of equivocation. For there is a need for raising the more basic point of order that the proponent used a contextually ambiguous sentence, without predicting anything about the outcome of the ensuing dialogue. 
Black makes an assertion by uttering sentence $S$. White can distinguish between more than one reading of $S$ and the utterance meaning machine does not rule out White's linguistic analysis.

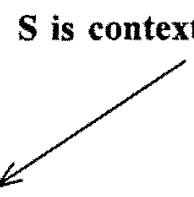

$S$ is not disqualified at the stage where Black makes an assertion by uttering $S$.

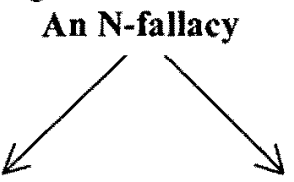

There is no disambiguation of Black's argument such that Black has an effective persuation strategy, although there is a disambiguation in which all reasons can be made acceptable and there is a disambiguation in which the warrant can be made acceptable.

An N-fallacy of ambiguity

\&

An N-fallacy of equivocation
In other cases.

N-fallacy
of
ambiguity

$S$ is disqualified at the stage where Black makes an assertion by uttering $\mathrm{S}$.

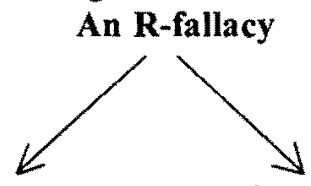

There is no disambiguation of Black's argument such that Black has an effective persuasion strategy, although there is a disambiguation in which all reasons can be made acceptable and there is a disambiguation in which the warrant can be made acceptable.

An R-fallacy of ambiguity

\&

An R-fallacy of equivocation
In other cases.

R-fallacy of ambiguity

Figure 3: Different types of fallacies 


\section{Appendix: Towards a formal model of ambiguity dialectics}

In this appendix I give an incomplete sketch of a formal model that states reasonable dialectical rights and obligations for discussants. The model $\mathrm{AD}$ is a close relative of the family of models for persuasion dialogues that is presented in Walton $\&$ Krabbe (1995). In the description of AD, I have made ample use of notations and ideas from these models. The models to be found there are richer in that they regulate incurring and retracting different kinds of commitments. $A D$ is richer in that it contains rules concerning the use and criticism of ambiguous sentences and in incorporating criticism of $\mathrm{N}$-fallacies.

$\mathrm{AD}$ has also features of pragma-dialectics, in that it makes use of the idea of a norm for critical discussion. Pragma-dialectics is richer in that it concerns more important norms for critical discussion. $\mathrm{AD}$ is richer in that it incorporates the norm for language use into a more permissive dialectical model in which fallacies or norm-violations can be discussed.

\section{Norms of discussion}

There is only one norm in $\mathrm{AD}$, the one stated in section 5 . To improve the model, other norms should be incorporated.

Norm: Do not make assertions by uttering sentences that are contextually ambiguous!

The norm has a strategic flavor, for what is or is not a contextually ambiguous sentence is, according to the present perspective, determined during the dialogue. If a sentence is or gets disqualified at a certain stage, while it is not reinstated at a later stage of the discussion as not-disqualified, then this sentence is at all stages of the discussion contextually ambiguous.

\section{Locution Rules of AD}

In the locution rules it is stated what language the discussants use, what speech acts they can perform with sentences of this language and what a move in an ADdiscussion looks like.

1: The language that is used in the discussion is the language of sentential logic, named $\mathrm{L}$. The set of sentences of $\mathrm{L}$ is defined by using an infinite set of atomic sentence letters, $P_{1}, P_{2}, P_{3}, \ldots$, the logical connectors, $\wedge, \vee, \neg, \rightarrow, \leftrightarrow$, and parentheses, ) and (. All atomic sentence letters are sentences of $L$. Suppose $S$ and $T$ are sentences of $L$, then so are $(S \wedge T),(S \vee T), \neg S,(S \rightarrow T)$ and $(S \leftrightarrow T)$. Nothing else is a sentence of $\mathrm{L}$. In order to refer to arbitrary sentences of $\mathrm{L}$, use will be made of the letters $\mathrm{S}, \mathrm{S}^{\prime}, \mathrm{T}, \mathrm{T}^{\prime}, \mathrm{S}_{1}, \mathrm{~S}_{2}, \ldots, \mathrm{T}_{1}, \mathrm{~T}_{2}, \ldots, \mathrm{S}_{1}^{\prime}, \mathrm{S}_{2}^{\prime}, \ldots$, $\mathrm{T}_{1}^{\prime}, \mathrm{T}_{2}^{\prime}, \ldots$ 
2: The following types of locutions or speech acts are permitted during the dialogue.

Elementary arguments:

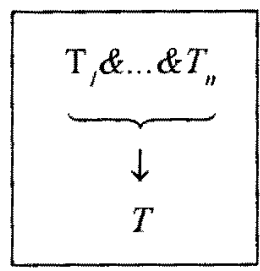

or equivalently, $T, \& \ldots \& T_{n} s o T$, or more shortly, $\Delta s o T$.

Disambiguated basic arguments at stage i: (see definition below)

Concessions:

$c(S)$

Challenges:

$S ?$ ?

Fallacy criticisms:

Ambiguity criticisms:

(S) $A m b\left(S_{p}, \ldots, S_{n}\right)$,

where $\mathrm{S}, \mathrm{S}_{1}, \ldots, \mathrm{S}_{\mathrm{n}}$ are atomic sentence letters.

Equivocation criticisms: (S)Amb $\left(S_{1}, \ldots, S_{n}\right)$ \& I'll win the ensuing dialogue, where $S, S_{1}, \ldots, S_{n}$ are atomic sentence letters.

Retractions: $n c(S)$, $n c\left((S) A m b\left(S_{p}, \ldots, S_{n}\right)\right)$, $n c\left((S) A m b\left(S_{1}, \ldots, S_{n}\right) \&\right.$ I'll win the ensuing dialogue $)$

By 'disambiguated basic argument at stage $i$ ' the following is meant. The rules of $\mathrm{AD}$ are such that White is allowed to challenge one of the elements $\mathrm{Q}$ of an elementary arguments $T_{1} \& \ldots \& T_{n} s o T$. The elements of an elementary argument are the reasons $T_{i}$ and the associated warrant $\left(T_{i} \wedge \ldots \wedge T_{i}\right) \rightarrow T$. After such a challenge Black has a prima facie obligation to offer an elementary argument for the challenged element. So, when at a stage Black has offered a few elementary arguments, they can be combined into one complex of elementary arguments. Such a complex is named a basic argument by Walton and Krabbe (1995, p. 129), and is defined by me in a slightly different way.

a. Elementary arguments $T, \& \ldots \& T_{n}$ soT are basic arguments.

b. If $\Delta$ is a basic argument with premises $T_{1}, \ldots, T_{j}, \ldots, T_{n}$ and conclusion $T$, and if $T^{\prime}{ }_{1}, \ldots, T_{m}^{\prime}$ are any sentences of $L$, then the configuration $\Delta^{\prime}$, which one gets by writing the following configuration of linked premises and a brace and arrow 


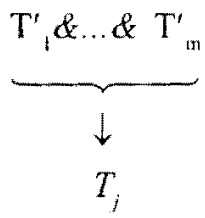

over $T_{j}$, or over the added associated warrant $\left(T_{1} \wedge \ldots \wedge T_{n}\right) \rightarrow T$ in the following manner

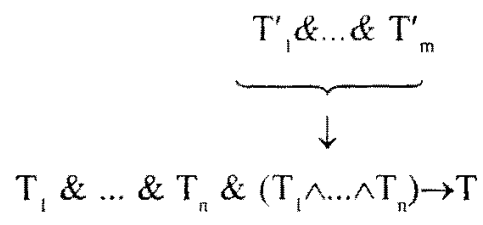

is again a basic argument.

c. Nothing is a basic argument that cannot be constructed by the above two rules.

If White criticizes Black of having used some contextually ambiguous atomic sentence, she does this by presenting the allegedly ambiguous atomic sentence $S$ and some disambiguating reformulations $S_{1}, \ldots, S_{n}$. A disambiguated basic argument at stage $i$ is formed by the basic argument, constituted by all elementary arguments that Black has offered until stage $i$, in which all occurrences of $S$ are replaced by occurrences of $S_{1}, \ldots, S_{n}$ or by occurrences of even other atomic sentences $\mathrm{S}_{1}^{\prime}, \ldots, \mathrm{S}_{\mathrm{mi}}^{\prime}$.

3: A move is made up of either:

- a challenge, possibly combined with some concessions, or

- an elementary argument, or

- a fallacy criticism, or

- an utterance meaning testing procedure, possibly combined with a disambiguated basic argument, or

- a disambiguated basic argument, or

- a retraction of a fallacy criticism, or

- a concession of the main thesis. possibly combined with a retraction of an equivocation criticism, or

- a retraction of the main thesis.

\section{Commitment Rules}

In order to enable the discussants to keep track of their obligations, the focal points of these obligations, sentences, are kept in accessible stores. The commitment rules determine what gets into and out of these stores. White can commit herself not only to topic sentences but also to the view that Black has violated a norm by using an ambiguous sentence $\mathrm{S}$. 
1: Each party has a commitment store, which indicates the party's obligations at a certain stage of the discussion. A commitment store is a set with sentences of L or fallacy criticisms as its elements.

2: At the start of the discussion Black's commitment store contains exactly one sentence, which is the main thesis throughout the discussion. White's commitment store may be empty, but may also contain sentences of L.

3: If White uses the expression $c(S)$ at a stage $\mathrm{i}$, then from the next stage on, White's commitment store also contains $S$.

4: If Black uses an elementary argument $T_{1} \& \ldots . T_{n}$ so $T$ at stage $\mathrm{i}$, then from stage $i+1$ Black's commitment store contains $T_{t}$ and $\ldots .$. and $T_{n}$, and also the warrant associated with the elementary argument $\left(T_{1} \wedge \ldots \wedge T_{n}\right) \rightarrow T$.

5: If White raises a fallacy criticism at stage i $(S) A m b\left(S_{,}, \ldots, S_{n}\right)$ or $(S) A m b\left(S_{1}, \ldots, S_{n}\right)$ \& I'll win the ensuing dialogue, then from the next stage on, White's commitment store contains this fallacy criticism.

6: If White raises a fallacy criticism against $S$, then $S$ is disqualified until White retracts this fallacy criticism.

7: If Black uses a disambiguated basic argument at stage i, then Black's commitment store is emptied and filled again with all basic reasons of the disambiguated basic argument together with all warrants associated with the elementary arguments that make up the disambiguated basic argument.

8: If a party retracts a sentence $S$ or a fallacy criticism $F$ at stage i, then from the next stage on, $S$ or $F$ is no longer an element of this party's commitments store,

\section{Structural Rules}

1: The parties make moves alternately.

2: White makes the first move in which she challenges Black's main thesis.

3: If in the preceding move White challenged Black's assertion $T$, then Black has to offer an elementary argument, $\Delta s o T$.

4: $\Delta$ so $T$ may be used only if some earlier move contained $T$ ??

5: If the preceding move contained an elementary argument $\Delta$ so $T$, White must either challenge one element $Q$ (a reason or a warrant) of the argument $\Delta s o T$, challenge one element $Q$ (a reason or a warrant) of the argument $\Delta s o T$ and concede some other elements of $\Delta s o T$, raise a fallacy criticism, or concede the main thesis.

6: A challenge $T_{i}$ ?? by White is allowed only if $T_{i}$ is not a concession of White, $T$, has not been challenged before, $T_{i}$ is in Black's commitment store and $T_{i}$ is an element of the argument offered by Black in the preceding move.

7: If $S$ is an atomic sentence that occurs within an argument offered by Black at stage $\mathrm{j}$, then White is allowed to utter $(S) A m b\left(S_{1}, \ldots, S_{\mathrm{n}}\right)$ or to utter $(S) A m b\left(S_{1}, \ldots, S_{n}\right)$ \& I'll win the ensuing dialogue at stage $\mathrm{j}+1$, unless $S$ has been proposed by White as a disambiguated reformulation in some fallacy criticism at stage $\mathrm{i}, \mathrm{i}<\mathrm{j}$.

8: If the preceding move contains $(S) A m b\left(S_{1}, \ldots, S_{\mathrm{n}}\right)$, or $(S) A m b\left(S_{1}, \ldots, S_{\mathrm{n}}\right) \&$ I'll win the ensuing dialogue then Black must either 
a) perform an utterance meaning testing procedure, that is, checking whether in this context of use each of the $S_{i}$ 's is a formulation of an interpretation of $S$ that is admissible from a linguistic point of view, or

b) offer a disambiguated basic argument. ${ }^{21}$

9: If the outcome of an utterance meaning testing procedure performed by Black is negative, then White must retract the fallacy criticism. If the outcome is positive, then Black is obliged to present a disambiguated basic argument in the same move.

10: If Black offers a disambiguated argument in which he uses disambiguated formulations $S^{\prime}, \ldots, S_{m}^{\prime}$, that were not proposed by White in her fallacy criticism (S) $A m b\left(S_{1}, \ldots, S_{n}\right)$ or in $(S) A m b\left(S_{p}, \ldots, S_{t}\right) \& I ' l l$ win the ensuing dialogue, then White may perform an utterance meaning testing procedure that has as an input the allegedly ambiguous sentence $S$, the set of disambiguated formulations $S_{1}, \ldots, S_{m}^{\prime}$ and the current context of use $C$. If the outcome is positive, Black repeats his disambiguated argument, if the outcome is negative, Black is obliged to offer a new disambiguated basic argument, in which all occurrences of $S$ are replaced by using only $S_{\imath}, \ldots, S_{n}$.

11: If an earlier stage contained an utterance meaning testing procedure with as input $S, S_{i}, \ldots, S_{n}$ and $\mathrm{C}$, then it is not allowed to perform this test with exactly this input again.

12: If Black uses some atomic sentence $S_{i}$ in a disambiguated basic argument and $S_{i}$ does not occur in a fallacy criticism by White, then White has the right to raise a fallacy criticism towards $S_{i}$.

13: If the preceding move contains an equivocation criticism, and Black offers a disambiguated basic argument, then this move is regarded as a challenge to the second conjunct of White's criticism, because by that Black starts a discussion, such that if White concedes the main thesis, she immediately has to retract her equivocation criticism.

14: If Black offers a disambiguated argument then White may challenge a basic reason of this complex argument, or one of the warrants that are associated with the elementary arguments that make up the disambiguated argument.

15: If White wins the discussion after she raised an equivocation criticism, then the equivocation criticism is regarded as sustained. ${ }^{22}$

16: If White raises a fallacy criticism at stage $i+1$ and retracts it at a later stage $\mathrm{j}$, then Black is allowed to repeat at stage $\mathrm{j}+\mathrm{l}$ his argument offered at stage $\mathrm{i}$.

17: If White has uttered an equivocation criticism, has not yet retracted it and utters $c(T)$, where $T$ is the main thesis, then she has to retract the equivocation criticism immediately.

18: It is not allowed to use disqualified sentences in elementary or disambiguated basic arguments.

19: At every stage, Black is allowed to utter $n c(T)$, where $T$ is the main thesis of the discussion. 
20: At every stage, White is allowed to utter $c(T)$, where $T$ is the main thesis of the discussion.

\section{Win-and-Loss Rules}

1: If Black utters $n c(T), T$ being the main thesis, then Black has lost the discussion, and White has won the discussion.

2: If White utters $c(T), T$ being the main thesis, then White has lost the discussion, and Black has won the discussion.

\section{Notes}

' Pragma-dialectics has much to say about applying the rules to real discussions, but this is not considered part of the ideal model for critical discussion. However, in 1984 and 2000 van Eemeren and Grootendorst propose a rule that states rights and obligations concerning requests for usage declaratives. These requests seem to be related to fallacy criticisms.

${ }^{2} \mathrm{Hamblin}$ named contributions that pertain directly to the issue under consideration topic points.

${ }^{3}$ The opponent may çriticize it by making a distinction between different readings of her own concessions. However, there is no real burden of proof associated with this criticism.

${ }^{4}$ Mackenzie, however, does not relate this to the theory of fallacies.

${ }^{5}$ In footnote 15 I shall give a motivation for this.

${ }^{6}$ The informal notion of the ambiguity of $S$ should not depend on the awareness or actual behavior of the opponent, but on something that can only be indicated vaguely: for instance, the opponent's actual behavior towards $S$ if she reflects thoroughly on her position or if her attention were directed to the need for making the distinction (M. Black 1990, 182). In the appendix, a more formal and restricted explication of ambiguity is given.

'To translate 'en presisering' by 'disambiguating reformulation' is a suggestion found in Barth and Krabbe, who themselves use the term 'clarifying reformulation' $(1982,21)$.

${ }^{8}$ This could be part of the shared background knowledge of the discussants; the discussants could have reached an agreement on it in the opening stage of the discussion; or the opponent could have expressed it during their present discussion. In the sketch of a formal model to be found in the appendix, I will only consider this last possibility.

${ }^{9}$ Although in such a case the proponent is aware of committing a fallacy of ambiguity.

${ }^{10}$ In such a case the proponent has an incorrect picture of the opponent's semantic dispositions, according to which the opponent sees just one reading, while the opponent actually sees a few readings but not the reading the proponent had in mind.

"The translation is mine. The first dialogue fragment below was constructed by me, the second dialogue fragment resembles to some degree what happened in court.

${ }^{12}$ This is a somewhat strong presupposition. It would suffice if the lawyer thinks there is a reasonable chance that this is an adequate picture of the judge's position.

${ }^{13}$ In van Eemeren \& Grootendorst (2000) there is a distinction between rules for critical discussion and rules of conduct. It seems especially to be directed to two different levels of generality of norms for critical discussion (as they are called in this paper).

${ }^{14}$ In order to make the model more complete, other norms should be incorporated.

15 The standard $\mathrm{AD}$-sequence is that the proponent offers an argument, and then the opponent does or does not concede the reasons. If the opponent made a concession that is ambiguous for the 
proponent, then the proponent can freely make use of a more precise formulated reason and see whether the opponent concedes or challenges it. So there is no urgent need for the proponent to criticize an ambiguous concession.

${ }^{16}$ The focus of an ambiguity criticism or equivocation criticism in $\mathrm{AD}$ is an atomic sentence $\mathrm{S}$, that may occur as a reason or thesis on its own, or as a constituent sentence of a logically more complex reason or thesis.

${ }^{17}$ Our brain resembles such a machine, except that the outcome of the machine is overt and decisive for the commitments of the discussants.

18 Using the pragma-dialectical notion of an opening stage this situation can be described as follows. The discussants have in the opening stage determined for some pairs of sentences, but not for all, that one of them is or is not a proper disambiguating reformulation of the other.

${ }^{19}$ Linguistic indeterminacy on the issue of synonymy will again lead to a positive outcome and will in this situation be in favor of Black.

${ }^{20}$ The points of order dealt with in this paper can be related to Krabbe's distinction in the following way. An ambiguity or equivocation criticism can be seen as an active criticism relative to the rules and as a fallacy criticism relative to the norm (Krabbe 1996, 134-135).

${ }^{21}$ This still leaves Black two options: using White's linguistic analysis or using an alternative analysis.

${ }^{22}$ In $\mathrm{AD}$ it is possible that an ambiguity criticism is retracted or sustained, after which the discussion continues. Raising this fallacy criticism has direct procedural consequences, as for whether some sentence $\mathrm{S}$ is disqualified or not. In $\mathrm{AD}$ it is not possible for the discussion to continue after an equivocation criticism has been sustained (but in some cases it is possible if it is retracted), for in a move in which it is sustained the discussion ends. However, the result of such an elementary kind of discussion is not only that party $\mathrm{X}$ won or lost the discussion, but also that, for at least these discussants, and for these kinds of issues, sentence $S$ is disqualified or not. The result of a successful equivocation criticism can have consequences for other, related discussions.

\section{References}

Barth, E.M. and E.C.W. Krabbe. 1982. From Axiom to Dialogue : A Philosophical Study of Logics and Argumentation. Berlin, New York: de Gruyter.

Black, M. 1990. Perplexities. New York: Cornell University Press.

Eemeren, F.H. van and R. Grootendorst. 1984. Speech acts in argumentative discussions: A theoretical model for the analysis of discussions directed towards solving conflicts of opinion. Dordrecht: Foris.

Eemeren, F.H. van and R. Grootendorst. 1992. Argumentation, Communication and Fallacies. Hillsdale N.J.: Lawrence Erlbaum.

Eemeren, F.H. van and R. Grootendorst. 2000. Kritische discussie, Amsterdam: Boom.

Finocchiaro, M.A. 1980. Galileo and the Art of Reasoning: Rhetorical Foundations of Logic and Scientific Method. Dordrecht: D. Reidel Publishing Company.

Hamblin, C.L. 1970. Fallacies. Virginia: Vale Press.

Krabbe, E.C.W. 1996. "Can we ever pin one down to a formal fallacy?." In J. van Benthem,

F.H. van Eemeren, R. Grootendorst and F. Veltman (eds.), Logic and Argumentation,

129-142. Amsterdam: Koninklijke Nederlandse Akademie van Wetenschappen.

Mackenzie, J.D. 1988. "Distinguo! The response to equivocation," Argumentation 2: $465-482$. 
Mackenzie, J.D. 1981. "The dialectics of Logic," Logique et analyse 24: 159-177.

Naess, A. 1966. Communication and Argument: Elements of applied semantics. Oslo: Universitetsforlaget.

Searle, J.R. 1970. Speech Acts: An essay in the philosophy of language. Cambridge: Cambridge University Press.

Walton, D. 1996. Fallacies Arising from Ambiguity. Dordrecht: Kluwer Academic Publishers.

Walton, D. and E.C.W. Krabbe. 1995. Commitment in Dialogue: Basic Concepts of Interpersonal Reasoning. New York: SUNY.

Jan Albert van Laar Filosofisch Instituut, Rijksuniversiteit Groningen A-weg 30, 9718 Groningen The Netherlands laar@philos.rug.n! 